\title{
AJAP1 expression modulates glioma cell motility and correlates with tumor growth and survival
}

\author{
CHUNHUI DI ${ }^{1}$, NIKOL MLADKOVA ${ }^{1}$, JAMES LIN $^{1}$, BRIAN FEE ${ }^{2}$, MIRIAM RIVAS $^{2}$, \\ KANG CHUNSHENG $^{3}$, DARELL BIGNER ${ }^{1,4}$ and DAVID CORY ADAMSON ${ }^{5,6}$
}

\author{
${ }^{1}$ Preston Robert Tisch Brain Tumor Center, Duke University Medical Center; ${ }^{2}$ Durham VA Medical Center, Durham, \\ NC, USA; ${ }^{3}$ Department of Neurosurgery, Tianjin Medical University General Hospital, Tianjin, P.R. China; \\ ${ }^{4}$ Department of Pathology, Duke University Medical Center, Durham, NC; ${ }^{5}$ Atlanta VA Medical Center, \\ Decatur, GA; ${ }^{6}$ Department of Neurosurgery, Emory University, Atlanta, GA, USA
}

Received July 15, 2017; Accepted September 21, 2017

DOI: 10.3892/ijo.2017.4184

\begin{abstract}
Glioblastoma multiforme (GBM) is one of the most common primary malignant brain tumors. Unraveling the molecular and genetic complexity that determines GBM's pronounced migratory property could provide new options for therapeutic targeting that may significantly complement current surgical and chemoradiation therapy and alter the current poor outcome. In this study, we establish stable AJAP1 overexpressing glioma cells in order to examine in vivo tumor growth. We examine AJAP1 localization by confocal microscopy and AJAP1's functional effect on migration and invasion across surfaces coated with laminin. Finally, analysis of AJAP1 expression in murine xenografts and GBM primary tumors revealed its association with tumor growth and survival. Stable overexpression of AJAP1 promotes adherence, decreases invasion of glioma cells through an extracellular-like matrix, and slows migration in the presence of laminin. These observations are reversed by gene knockdown using multiple siRNAs. Additionally, overexpression of AJAP1 decreases colony formation in glioma cells, and leads to smaller tumor growth with increased survival in glioma xenograft mice. Loss of AJAP1 protein expression predicts worse survival in GBM patients. AJAP1 overexpression decreases cell motility in the presence of laminin and decreases tumor growth in xenografts. Its loss of expression predicts worse survival in patients. This study extends our prior observations and implicates AJAP1 as a potential prognostic marker and a viable target for therapeutic intervention in GBM.
\end{abstract}

Correspondence to: Dr David Cory Adamson, Atlanta VA Medical Center Neurosurgery, 1670 Clairmont Road, Decatur, GA 30033 , USA

E-mail: cory.adamson@emory.edu

Key words: adherens junctions-associated protein, Shrew1, GBM, glioblastoma, malignant glioma, gene knockdown, gene overexpression, survival, migration, proliferation

\section{Introduction}

Glioblastoma multiforme (GBM) is one of the most common types of primary intracranial tumors that occur in adults (1), accounting for up to $30 \%$ of all brain tumors. As GBM is a malignant glioma, the prognosis for this disease remains universally dismal, in spite of modern diagnostics, sophisticated surgical techniques, and modern chemoradiotherapies. The median survival of GBM patients treated with the current standard of care, consisting of $>98 \%$ tumor mass removal followed by concurrent radiation treatment and chemotherapy with the alkylating agent temozolomide, only reaches 14 (13-16) months (2). The clinical outcome of GBM patients has improved only marginally during the past few decades of intensive investigation in all therapeutic modalities, surgery, chemotherapy, radiotherapy, and immunotherapies (3). Surgical treatment alone is able to provide only approximately 8 months of survival to the patients due to the ability of GBM tumor cells to rapidly infiltrate the neuropil and to migrate, even to sites distant from the original tumor focus. Given the short median survival for GBM patients who have been treated by multimodal approaches (4), any improvement in the clinical outcome for patients with new effective therapeutic options could greatly affect survival.

The resistance of GBM to standard therapy may be due to the multitude of heterogeneous genetic alterations related to glioma cell migration. Genetic studies of the greatest impact have consistently demonstrated a few altered signaling pathways that appear fundamentally related to GBM tumorigenesis, namely, activating events in the Akt, PI3K, Ras, PTEN, or receptor tyrosine kinase pathways (such as VEGFR, EGFR, and PDGFR) and decreased activity in the RB1, p16, or TP53 signaling cascades $(5,6)$. Some of these pathways have been implicated in the ability of cells to migrate away from the original tumor focus and diffusely invade normal cerebral parenchyma; however, no single pathway or factor appears central to this process. When untreated, more than $50 \%$ of these tumors will migrate to and invade the contralateral hemisphere (7). Inhibition of this infiltrative process could greatly enhance the effect of adjunctive therapies in the current standard of care. 
The invasive and migratory process of glioma cells is a complex, dynamic, and multifactorial process, involving multiple, discrete, but presumably coordinated steps, with alterations in intracellular signaling cascades and cell surface receptors that interact with cells and extracellular matrix (ECM) factors $(8,9)$. In GBM, various molecules have been implicated in these processes, such as ECM components, integrins, cell-cell interacting molecules such as cadherin, connexin 43, matrix metalloproteinases, Rho, Rac, FAK, and PYK2 $(8,9)$. However, the identification of these factors has not led to therapeutic interventions that have impacted the current clinical course of GBM.

We and others recently identified altered expression of AJAP1 in GBM, suggesting a potential role as a prognostic factor in GBM tumorigenesis $(10,11)$. Previously, using multiple genome-wide studies, we found that AJAPI is deleted in up to $16 \%$ of GBM patients and its expression lost in $86-92 \%$ of GBM patients (10). AJAP1's loss of expression correlates with promoter hypermethylation (10). AJAP1 was originally described as a small single transmembrane protein interacting with E-cadherin- $\beta$-catenin complexes in adherens junctions (12). Later, AJAP1 was shown to be deleted or at least expressed at low levels in oligodendroglioma (13) and neuroblastoma (14). Loss of AJAP1 expression has been associated with decreased invasion in HeLa cells (15). However, its restoration of expression has been suggested to decrease invasion in glioma cells (13). These studies suggest that AJAP1 may modulate migration, but may function differently in different cell types and environments. In this study, we extend our prior observations of AJAP1's effect on glioma motility by stably overexpressing it in glioma cells in the presence of laminin, but more importantly in an in vivo model. We also extend our prior observations of national clinical databases to our own more characterized institutional patient cohort to further establish its clinical relevance.

\section{Materials and methods}

Tumor samples and cells. Tumor samples ( $>95 \%$ pure tumor) were obtained in accordance with an IRB-approved protocol from the Tissue Biorepository of the Preston Robert Tisch Brain Tumor Center at Duke. Normal brain tissue from patients without brain tumors was obtained at the time of autopsy and frozen at $-80^{\circ} \mathrm{C}$ before DNA and RNA isolation. All glioma cell lines were provided by the Duke Tissue Culture Facility. Cell lines were maintained in DMEM Medium (Invitrogen, Carlsbad, CA, USA) supplemented with $10 \% \mathrm{FBS}$ at $37^{\circ} \mathrm{C}, 5 \%$ $\mathrm{CO}_{2}$, without antibiotics.

RNA extraction, cDNA synthesis, and quantitative PCR. Total RNA was isolated by using an RNeasy Mini kit (Qiagen, Valencia, CA, USA). Next, 200 ng of total RNA was reversetranscribed by using an iScript cDNA Synthesis kit (Bio-Rad, Hercules, CA, USA). Amplification of cDNA was monitored by following a SYBR Green protocol (Sigma, St. Louis, MO, USA) with a 7900HT Fast Real-time PCR System (Applied Biosystems, Foster City, CA, USA). PCR was initiated with a first denaturation step at $95^{\circ} \mathrm{C}$ for $10 \mathrm{~min}$, followed by 40 cycles at $95^{\circ} \mathrm{C}$ for $15 \mathrm{sec}$ and $60^{\circ} \mathrm{C}$ for $1 \mathrm{~min}$. To avoid amplification of contaminated genomic DNA, forward and reverse primers were designed at different exons (AJAP1 forward: GTTA GCACAACGGAGCCTTC, reverse: CTTTGGGCACAGCA ATTTTT; GAPDH forward: GAAGGTGAAGGTCGGA GTCA, reverse: AATTTGCCATGGGTGGAAT). Quantitative values were obtained from the threshold cycle $(\mathrm{Ct})$ number at which the increase in the signal associated with exponential growth of PCR products could first be detected by using SDS2.2.2 software (Applied Biosystems). The transcript level of AJAP1 gene was normalized to that of $G A P D H$. As a reference, we used adult human brain RNA (BioChain Institute, Hayward, CA, USA).

Stable overexpression studies. The AJAPI cDNA was isolated from normal cortex by a PCR approach and ligated into the PCR-Blunt vector (Invitrogen). The sequence was verified at the Duke DNA Sequencing Facility and confirmed by NCBI Blast. The AJAPl cDNA was removed with KpnI and HindIII restriction enzymes and subcloned into the pEGFP-N1 expression plasmid. The pEGFP-AJAP1 and pEGFP-N1 (control) expression plasmids were transfected with Lipofectamine 2000 (Invitrogen) into glioma cells. After $24 \mathrm{~h}$, cells were diluted by 1:100 and grown in 10\% DMEM containing $0.5 \mathrm{mg} / \mathrm{ml}$ of G418. One week later, cells were diluted by 1:10,000 again and cultured in 10\% DMEM containing $0.5 \mathrm{mg}$ of G418. After 2-3 weeks, multiple clones from single cells with green fluorescence were transferred to 6-well plates, grown, and tested for best expression by fluorescent microscopy and western blotting.

AJAP1 knockdown studies. Two different siRNA oligonucleotides for AJAP1 inhibition (AJAP1 Stealth RNAi siRNA HSS148198 and AJAP1 Stealth RNAi siRNA HSS148199, Invitrogen) and scrambled siRNA for the negative control were obtained (Invitrogen). A total of $2 \times 10^{5}$ cells were seeded in 6-well plates and grown to 50-60\% confluency in $1.5 \mathrm{ml}$ of culture medium before siRNA transfection. Cells were washed with PBS three times before transfection. Cells were transfected by adding Lipofectamine 2000 transfection reagent (Invitrogen) and siRNA dissolved in Opti-MEM I Reduced Serum Medium without FBS (0.5 nmol/1 per well) according to manufacturer's recommendations. Cells were maintained in transfection medium overnight and subsequently in culture medium for 24,48 , and $72 \mathrm{~h}$ before analysis. The extent of RNA knockdown under these conditions was determined by cDNA synthesis as previously described and visualization on agarose gel. At $48 \mathrm{~h}$, the loss of AJAP1 was optimal, so this time was selected for procedures and applied in all knockdown experiments.

Cell proliferation. Cell proliferation was assessed by 3-(4,5-dimethylthiazol-2-yl)-2,5-diphenyltetrazolium bromide (MTT) assay. Briefly, following the appropriate experiment, cells were trypsinized and seeded at a density of $1 \times 10^{3}$ per well in a 96-well plate $48 \mathrm{~h}$ after siRNA transfection. At various time-points, culture medium was replaced by $200 \mu \mathrm{l}$ of MTT solution $(0.5 \mathrm{mg} / \mathrm{ml})$ and incubated for $4 \mathrm{~h}$ at $37^{\circ} \mathrm{C}$ and $5 \%$ $\mathrm{CO}_{2}$. Once the incubation was terminated and the presence of purple formazan crystals was verified by light microscopy, MTT solution was discarded and $200 \mu \mathrm{l}$ of DMSO was added to dissolve the formazan crystals. Absorbance was measured 
at $570 \mathrm{~nm}$ with an ELISA reader, and 8 to 12 wells were evaluated for each experimental condition.

Adhesion assay. Cell adhesion to ECM protein was measured. The 6-well plates were covered by $2 \mathrm{ml}$ of PBS with laminin at a 1:1,000 ratio (a kind gift of Dr Irina Lebedeva from the Department of Neurobiology at Duke) for $1 \mathrm{~h}$ at room temperature. Cells from each experiment were then resuspended so that $1 \times 10^{5}$ cells were included in $2 \mathrm{ml}$ of medium, which was added to each well, then incubated for $1 \mathrm{~h}$ at $37^{\circ} \mathrm{C}$ and $5 \%$ $\mathrm{CO}_{2}$. The experiment was performed in triplicate. After $1 \mathrm{~h}$, the medium with unattached cells was removed, and wells were washed with PBS three times. Adherent cells were stained with $0.5 \%$ crystal violent and dissolved in $0.5 \mathrm{ml}$ of $33 \%$ acetic acid per well. The absorbance was then measured at $595 \mathrm{~nm}$.

Transwell invasion assay. Transwell cell invasion assays were performed by using modified Boyden chambers (Transwell; Corning Inc., Corning, NY, USA). In brief, cells were cultured overnight in FBS-free medium, then trypsinized, washed, and suspended in serum-free medium at $10^{6}$ cells $/ \mathrm{ml}$. Next, $250 \mathrm{Ml}$ of cells was placed into the upper chambers with and without Matrigel and followed for various time points (2-24 h). In the bottom well, medium with $10 \%$ FBS was added to induce chemoattraction for the cells. Cells that migrated to the underside of the chamber were fixed with $3.7 \%$ paraformaldehyde in PBS. After cells were removed from the upper side of the chamber, the membranes were removed and stained with $0.5 \%$ crystal violent. Cells that invaded through the Matrigel onto the undersurface of the membrane were photographed and hand-counted by light microscopy, as well as optically densitized at $595 \mathrm{~nm}$ by an ELISA plate reader.

Wound healing migration assay. Cell migration was also analyzed by using a wound healing assay. Briefly, cells were seeded at a density of $2 \times 10^{5}$ per well in a 6 -well plate. At the appropriate times, cell monolayers were scratched with the tip of a standard 200- $\mu$ l pipette tip. After being washed three times with PBS, cells were microphotographed (x400) and then incubated under standard conditions. Migration into the scratched area was documented at various time-points: 6, 12, 24 , and $48 \mathrm{~h}$ after wounding. Scratch closure by migrating cells was compared in the experimental and the control cells relative to the total area of the scratches. Three to six scratches were evaluated for each experimental condition.

Western blot studies. Cells were lysed in RIPA buffer (Pierce, Rockford, IL), and protein was quantified with Coomassie Blue protein standards (Pierce). Lysed cells were mixed 1:1 with Laemmli buffer and boiled for $5 \mathrm{~min}$, and then 25-50 $\mu \mathrm{g}$ protein for each sample was equally added to individual lanes and electrophoresed onto agarose gels (NuPage, Invitrogen) for $\sim 35 \mathrm{~min}$ at $186 \mathrm{~V}$. Protein was transferred from gels to nitrocellulose membranes by semi-dry transfer cell (Bio-Rad) for $\sim 30 \mathrm{~min}$ at $12-15 \mathrm{~V}$. Membrane was blocked for $30 \mathrm{~min}$ in 5\% nonfat dry milk TBST buffer, washed, and incubated with primary antibody at room temperature for 1-2 $\mathrm{h}$. After washing 3 times with TBST buffer, membrane was incubated with secondary antibody HRP conjugated to IgG at room temperature for $1 \mathrm{~h}$. Proteins were detected with Supersignal West Femto Substrate (Pierce).

Glioma xenografts. Mice $(\mathrm{n}=10)$ were anesthetized i.p. with a $0.3-\mathrm{ml}$ Ketamin and xylazine saline (1:10) mixture per approved animal protocol. Once the mice were sedated, they were placed in a stereotactic frame and secured with ear bars to the frame. Next, $1 \times 10^{6}$ cells in $10 \mu \mathrm{l}$ PBS were injected into each mouse brain, 1-mm lateral to bregma, 2-mm anterior to coronal suture, and 3-mm deep. A total of $1 \mu 1$ was injected over a 10 -min period with a 1-min interval between each injection. Mice were marked after successful injections and immediately returned to their cage for direct observation until fully recovered from anesthesia, typically within $20 \mathrm{~min}$. After completion of the study, brains were harvested en bloc, immediately submerged in $10 \%$ formalin for $24 \mathrm{~h}$, and then paraffin-embedded.

Immunohistochemistry. For immunohistochemical analysis, 4- $\mu \mathrm{m}$ sections were deparaffinized with xylene three times, washed with $100 \%$ ethanol to remove xylene, and rehydrated in a graded series of ethanol. Slides were placed in a boiling water bath for $20 \mathrm{~min}$ and then cooled at room temperature for $30 \mathrm{~min}$. Slides were washed with water and treated with $0.3 \%$ hydrogen peroxide in methanol for $10 \mathrm{~min}$. Excess moisture was removed, and tissues were encircled by ImmEdge pen (Vector Laboratories, Burlingame, CA, USA). Slides were washed in PBS and then blocked for $1 \mathrm{~h}$ with blocking buffer (PBS $+1 \%$ BSA $+20 \%$ horse serum). Block solution was aspirated away and primary antibody $(4 \mu \mathrm{g} / \mathrm{ml})$ applied in a humidity chamber at $4^{\circ} \mathrm{C}$ overnight. After a PBS wash, secondary mouse antibody (1:100 diluted, Vector Laboratories) was incubated on slides for $1 \mathrm{~h}$ at room temperature, followed by PBS wash. ABC Standard Elite kit (Vector Laboratories, PK-6100) solution was applied to the slides for $30 \mathrm{~min}$ at room temperature and washed away with PBS. DAB reagent was applied to develop the slides, which were then washed with PBS, counterstained with standard H\&E, and mounted with Cytoseal XYL (Richard-Allan Scientific, Kalamazoo, MI, USA).

Statistical analysis. Statistical significance was set a priori at $\mathrm{P}<0.05$. Analyses were performed with Microsoft Excel and SAS E-guide statistical packages (SAS, Inc., Cary, NC, USA).

\section{Results}

AJAP1 localizes to the plasma membrane in glioma cells. We previously demonstrated frequent and marked reduction in expression of AJAP1 in a large cohort of primary tumors and glioma cell lines when compared to normal brain (10). AJAP1 protein expression was absent or markedly reduced in the majority of glioma cell lines tested. In our prior study, we demonstrated that targeted knock down of endogenous AJAP1 in D409 could increase motility characteristics. In this study, we chose a glioma cell line with no AJAP1 expression (U87; data not shown) and one with markedly reduced expression (U373; data not shown) to evaluate AJAP1's effect on motility in the presence of a common extracellular matrix (ECM) factor and more importantly, evaluate its effect in vivo. After subcloning the AJAP1 cDNA into the pEGFP-N1 expression plasmid and 
A

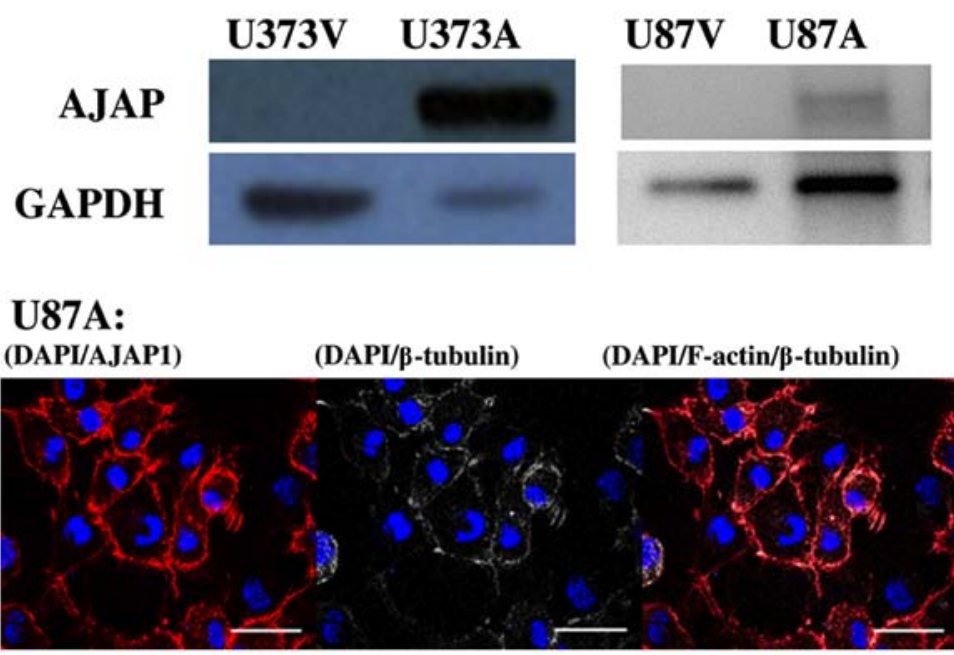

Figure 1. AJAP1 is targeted to plasma membranes in glioma cells. When AJAP1 is stably overexpressed in U87 and U373 glioma cells with pEGFP-AJAP1 [(A) endogenous markedly reduced expression of AJAP1 in U373 (data not shown) is smaller molecular weight than the AJAP1-GFP fusion protein and not reflected on this blot], it is consistently targeted to plasma membranes [(B) DAPI is blue, AJAP1 is red in left panel, $\beta$-tubulin is white, and F-actin is red in the right panel]. U373V, empty plasmid; U373A, AJAP1 plasmid; U87V, empty plasmid; U87A, AJAP1 plasmid. F-actin and $\beta$-tubulin are also labeled to help outline cellular morphology. Bar, $10 \mu \mathrm{m}$.
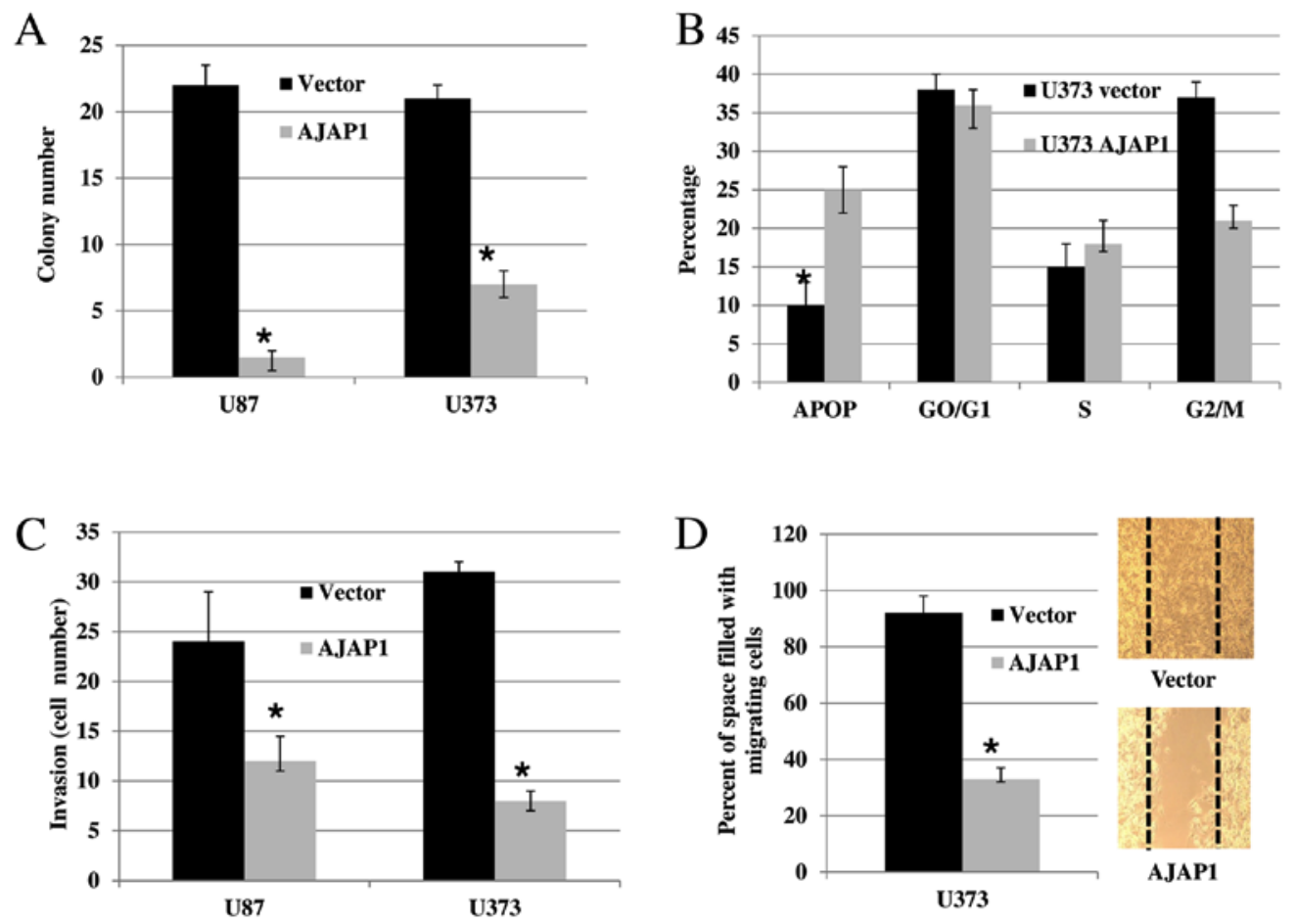

Figure 2. AJAP1 overexpression decreases glioma cell colony formation, increases apoptosis, and decreases invasion and migration. Overexpression of AJAP1 in U87 and U373 resulted in significantly fewer colonies after growth in soft agar for 2 weeks (A). Cell cycle FACS analysis of U373 that overexpresses AJAP1 suggests that apoptotic cell death is increased, whereas cells without AJAP1 have a lower apoptotic cell death rate [(B) G0/G1, S, G2/M are cell cycle stages]. Glioma cells U87 and U373 invade through a Matrigel matrix and 8- $\mu \mathrm{m}$ membrane pore at a significantly lower rate when AJAP1 is overexpressed on their surface (C). U373 cells migrate across a surface significantly less quickly with AJAP1 present (D). Experiments done in triplicate. "P<0.05.

stably transfecting the plasmid into glioma cells, we confirmed robust AJAP1 expression by immunoblot (Fig. 1A; endogenous markedly reduced expression of AJAP1 in U373 is smaller molecular weight than the AJAP1-GFP fusion protein and not reflected on this blot). Confocal microscopy confirmed AJAP1 was consistently overexpressed on the plasma membranes of glioma cells (Fig. 1B; red-tagged AJAP1 in left panel; F-actin and $\beta$-tubulin are also labeled to help outline cellular morphology in middle and right panel). We also transfected non-glioma neoplastic (MCF7) and non-neoplastic (RK3E) cells to demonstrate consistent membrane localization in other cell types (data not shown). We looked at MCF7 as a positive control, as previously seen by others $(16,17)$. This suggests stable expression of an intact AJAP1 protein that localizes to the plasmid membrane in glioma cells. Protein analysis based on amino acid sequence also suggests a single transmembrane 
A

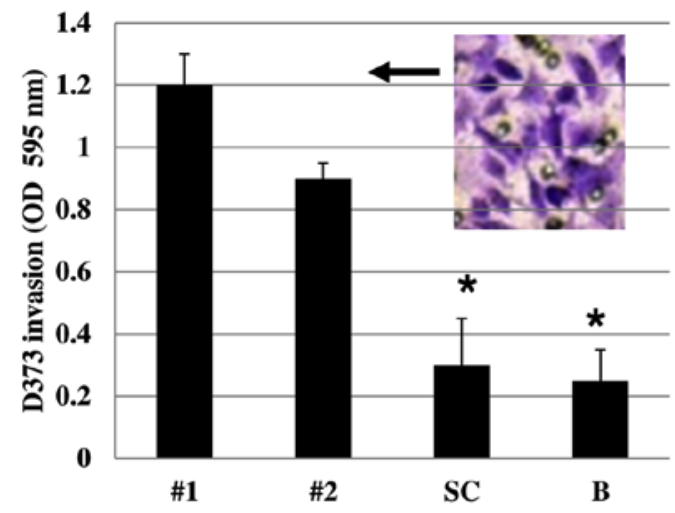

$\mathrm{C}$

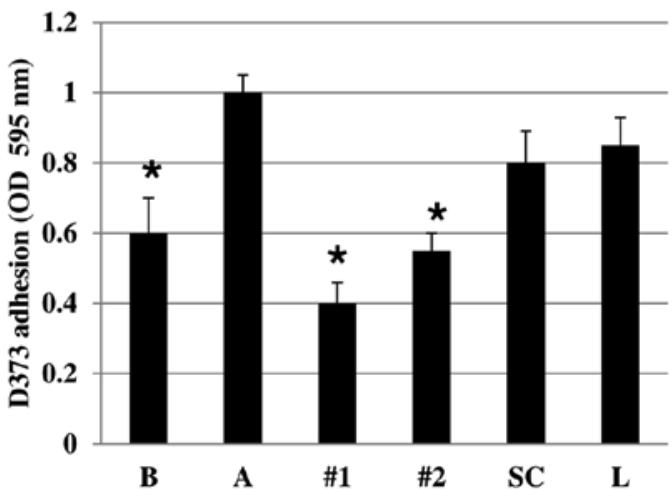

B

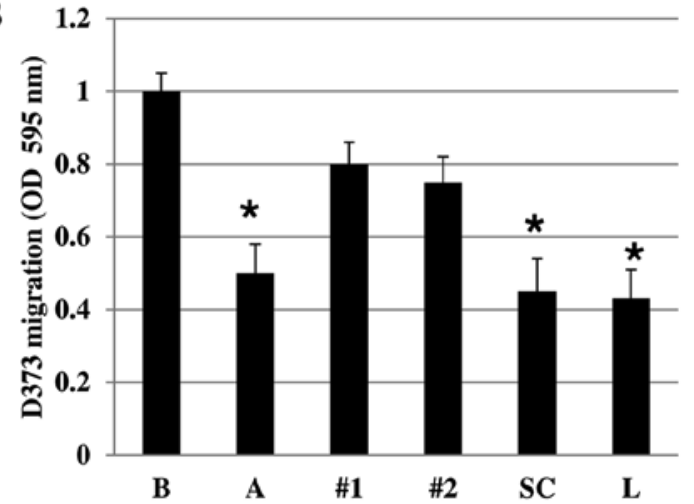

$\mathrm{D}$

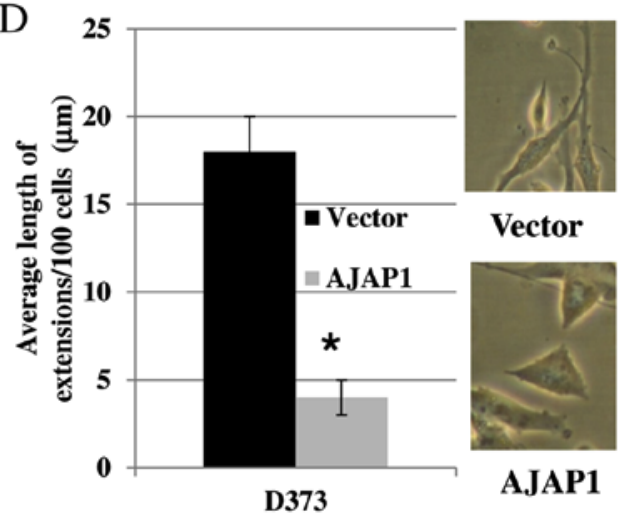

Figure 3. Loss of AJAP1 expression by siRNA knockdown increases glioma cell adherence, invasion, and migration. More D373 glioma cells invaded through Matrigel Transwell assays when AJAP1 is lost by siRNA (A; siRNA\#1, siRNA\#2), as compared to scrambled siRNA (SC) or untreated cells (B=baseline). Insert illustrates representative membrane with cells stained blue, $\mathrm{x} 40$ ). D373 cells that have been engineered to stably overexpress AJAP1 migrate less ( $\mathrm{A}=\mathrm{AJAP} 1$ compared to $\mathrm{B}=$ baseline), but show increased migration after gene knockdown [\#1 and \#2 compared to scrambled (SC) siRNA or lipofectamine only (L) (B)]. Significantly fewer D373 cells that stably overexpressed AJAP1 adhered when AJAP1 was knocked down (C). D373 cells are larger, are more amoeboid-shaped, and have longer and larger extensions when AJAP1 is knocked down (D). Baseline, cells alone; A=AJAP1 stably transfected cells; \#1, AJAP1 stable transfected cells subsequently treated with siRNA\#1; SC, AJAP1 stably transfected cells subsequently treated with scrambled siRNA; lipo, Lipofectamine only. Experiments done in triplicate. ${ }^{*} \mathrm{P}<0.05$.

domain with a long N-terminus extracellular domain with targeting sequence characteristics that have been studied by others in epithelial cells (18-20).

AJAP1 overexpression decreases glioma cell colony formation, increases apoptosis, and decreases invasion and migration. Stable AJAP1 overexpression did not significantly alter cell proliferation in our glioma cell lines, even after serum starvation (data not shown). However, overexpression of AJAP1 in U87 and U373 resulted in fewer colonies after growth in soft agar for 2 weeks, an assay measuring cell anchorage-independent growth (Fig. 2A). Under normal physiologic conditions, cell death is essential for the maintenance of appropriate cell number. However, the rate of cell death may be disturbed in tumorigenesis. Cell cycle FACS analysis of U373 that overexpressed AJAP1 suggests that apoptotic cell death was increased (Fig. 2B). Cells without AJAP1 had a lower apoptotic cell death rate. Glioma cells invaded through a Matrigel matrix and $8-\mu \mathrm{m}$ membrane pore at a significantly lower rate when AJAP1 was overexpressed on their surface (Fig. 2C), suggesting less ability to invade ECM-like material when AJAP1 is expressed. Migration of glioma cells across a surface in a wound healing assay was significantly less with AJAP1 present (Fig. 2D).
Loss of AJAPl expression by siRNA knockdown increases glioma cell invasion and migration. To ascertain the effect of the targeted loss of AJAP1 expression in glioma cells, we selected a glioma cell line with AJAP1 stably overexpressed (D373; Figs. 1 and 3) and knocked down expression with two AJAP1-specific siRNAs. When AJAP1 was reduced by two siRNAs (Fig. 3A\#1 and \#2), more glioma cells invaded through Matrigel Transwell assays, in comparison to scrambled siRNA (SC) or untreated baseline cells (B). Migration was reduced in glioma cells that were engineered to stably overexpress AJAP1 [Fig. 3B, baseline (B) vs. overexpressed AJAP1 (A)], but was increased after AJAP1 was knocked down (Fig. 3B \#1 and \#2). Similarly, significantly fewer glioma cells that stably overexpressed AJAP1 adhered than when AJAP1 was knocked down (Fig. 3C). D373 cells are larger, more amoeboid-shaped, and have longer and larger extensions when AJAP1 is knocked down (Fig. 3D).

AJAPl overexpression slows tumor growth and increases survival in mice. To evaluate the role of AJAP1 in vivo, mice ( $n=10 /$ group) were stereotactically injected intracranially with U87 glioma cells stably expressing the pEGFP-N1 or pEGFP-AJAP1 plasmid. As seen by immunohistochemistry (Fig. 4A, top left panel, whole mount), a typical injection 
A
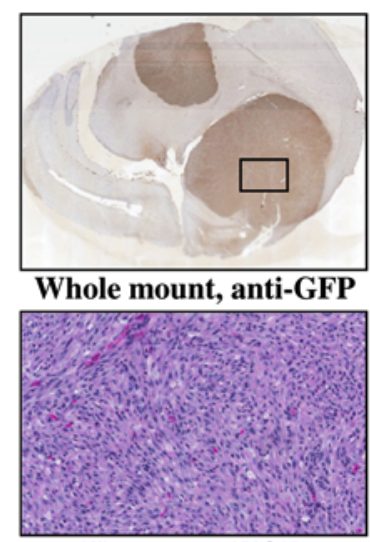

Vector $\mathrm{x5}, \mathrm{H \& E}$

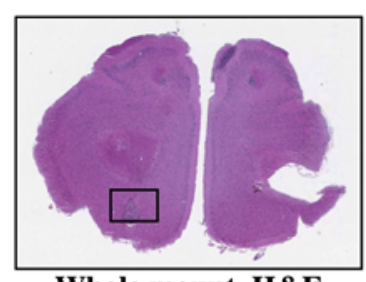

Whole mount, H\&E

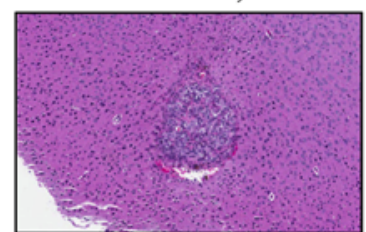

AJAP1 x5, H\&E
C

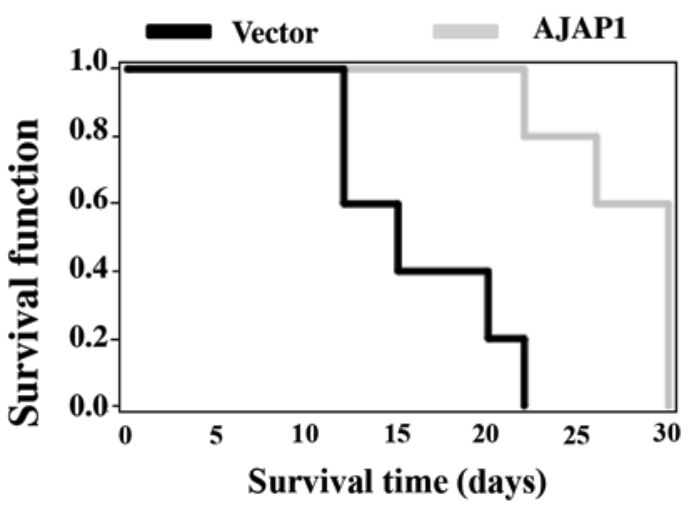

B

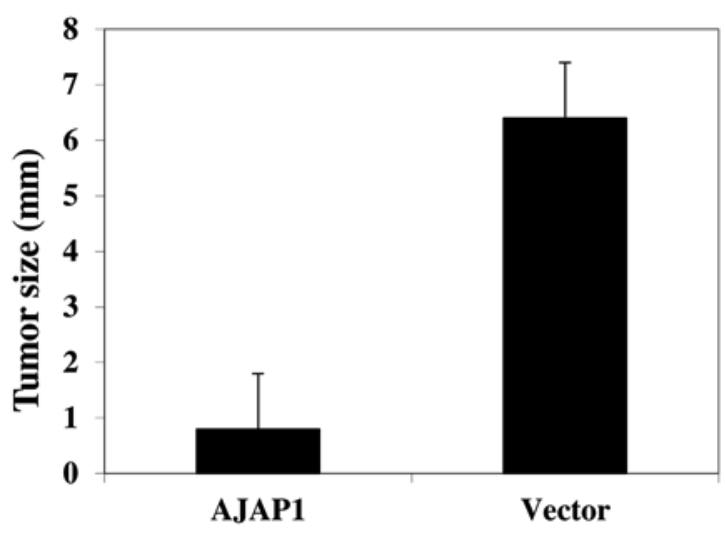

D

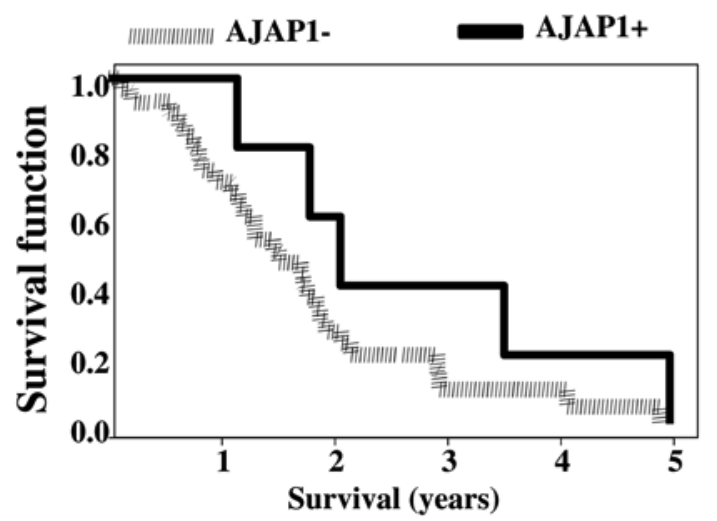

Figure 4. AJAP1 overexpression slows tumor growth and increases survival in mice. Murine xenografts (n=10/group) stereotactically injected intracranially with U87 glioma cells stably expressing the pEGFP-N1 plasmid as seen by immunohistochemistry (A, top left panel, whole mount showing two large tumor nodules positive for GFP). At high magnification, U87 tumors demonstrate increased cellularity, cellular and nuclear atypia, increased mitotic images, pseudopallisading cells, and hypervascularity (A, bottom left panel). U87 tumors with AJAP1 overexpression are significantly smaller (A, top right and bottom right panels). U87 gliomas that overexpress AJAP1 (B, AJAP1=pEGFP-AJAP1) result in significantly smaller tumors than AJAP1-negative tumor cells (B, Vector=pEGFP-N1). Glioma xenografts that overexpressed AJAP1 resulted in longer survival than AJAP1-negative cells. We evaluated a U87 xenograft with a pEGFP-N1 vector versus U87 xenograft with AJAP1 overexpression via a pEGFP-AJAP1 construct $(\mathrm{C}, \log$-rank $\mathrm{P}=0.01)$. AJAP1 loss demonstrates worse survival in GBM patients (D). Patients ( $\mathrm{n}=35$ ) with markedly reduced or absent AJAP1 (AJAP1-) protein expression trended toward worse survival than patients with intermediate or normal AJAP1 expression (AJAP1+) (log-rank $\mathrm{P}=0.09$ ). All patients underwent standard of care therapy, including gross total resection of visible enhancing tumor on MRI, followed by at least 6 months of concurrent radiation therapy and temozolomide. In this cohort, age $<45$ years predicted better survival $(\mathrm{P}<0.05)$. This is consistent with our prior observation of the public database Rembrandt, where all glioma patients were assessed Those with downregulation of AJAP1 expression ( $>2$-fold loss, $n=206$ ) clearly had significantly worse survival than those with intermediate or normal expres$\operatorname{sion}(\mathrm{n}=91 ; \mathrm{P}=0.0056)(10)$.

generated 1-2 large tumor nodules positive for GFP. At high magnification, tumors demonstrate typical characteristics of gliomagenesis including increased cellularity, cellular and nuclear atypia, increased mitotic figures, pseudopallisading cells, and hypervascularity (Fig. 4A, bottom left panel). Tumors with AJAP1 overexpression are significantly smaller than AJAP1 negative tumors (Fig. 4A, top right panel, and Fig. 4B). Glioma xenografts that overexpressed AJAP1 resulted in longer survival than AJAP1-negative cells. We evaluated a U87 xenograft with a pEGFP-N1 vector versus U87 xenograph with AJAP1 overexpression via a pEGFP-AJAP1 construct (Fig. 4C, log-rank $\mathrm{P}=0.01$ ).

AJAP1 loss demonstrates worse survival in GBM patients. Previously, using four independent sets of genomic data and human tumor samples, we found that the unique loss of AJAP1 is a frequent event in up to $16 \%$ of GBM primary tumors (10). In this study, we obtained linked clinical data from 35 adult GBM patients with measured protein expression of AJAP1.
All patients underwent standard of care therapy, including gross total resection of visible enhancing tumor $(>95 \%$ on postoperative MRI), followed by at least 6 months of concurrent radiation therapy and temozolomide. In this cohort, age $<45$ years predicted better survival $(\mathrm{P}<0.05)$, but age was not correlated with AJAP1 expression ( $\mathrm{P}>0.1)$. Patients with markedly reduced or absent AJAP1 expression clearly trended toward worse survival than patients with intermediate or normal AJAP1 expression (Fig. 4D). This is consistent with our prior observation of the public database Rembrandt, where all glioma patients were assessed (10). Those with downregulation of AJAP1 expression ( $>2$-fold loss, $n=206$ ) clearly had significantly worse survival than those with intermediate or normal expression $(\mathrm{n}=91 ; \mathrm{P}=0.0056)$ (10).

\section{Discussion}

Despite surgical removal of the visible tumor, GBM universally recurs because of the high migratory potential of tumor 
cells into nearby brain parenchyma (3). Determining how to effectively target critical signaling pathways or mediators of specific tumor cell features such as migration is of paramount importance for treating this disease.

In our prior study, we used digital karyotyping, microarrays and the TCGA dataset to investigate chromosome $1 \mathrm{p} 36$, a hotspot for genetic loss in cancers (10). With high-resolution mapping, we and others have demonstrated the frequent loss of AJAP1 expression in GBM primary tumors $(10,11)$. Others have demonstrated the loss of expression of AJAP1 in neuroblastomas (14), oligodendrogliomas (13), and ependymomas (21). We discovered genomic deletion of AJAP1 in up to $16 \%$ of these tumors and cell lines; however, a much larger percentage (86-92\%) had loss of expression of this gene.

Epigenetic silencing via cytosine methylation is a well-established and extensively used mechanism for gene regulation in numerous cancers, including GBM. Using mutational and methylation analyses, we previously showed that lack of AJAP1 expression is not due to promoter or gene mutation but is epigenetically silenced by promoter hypermethylation (10). Cogdell et al recently demonstrated increased methylation that correlated with less AJAP1 expression in oligodendroglioma (22). In our large series of primary GBM tumors and cell lines, 65 and 90\%, respectively, showed widespread evidence of AJAP1 methylation. Targeting methylated genes in GBM may be a viable option since demethylating agents have shown efficacy in preclinical studies (10) and clinical trials.

In this study, we have further explored the expression of AJAP1 in a large panel of glioma cells to identify cells with absent or low expression. After establishing glioma cells that stably overexpress AJAP1 on the plasma membrane, we provide ample evidence that modulating gene expression clearly affects tumor cell invasion and migration. Loss of AJAP1 resulted in increased invasion and migration. We were able to reverse the phenotype by knocking down the gene in cells that overexpress it. In addition, AJAP1 expression clearly affects cellular morphology. Glioma cells with the loss of AJAP1 took on a more amoeboid shape with extensive extensions, which suggests that these cells were actively exploring their environment to find a more suitable place to which to migrate and proliferate. In our xenograft tumor models, overexpression of AJAP1 markedly slowed tumor growth and increased survival. Finally, we demonstrate a clear association of the loss of AJAP1 expression and worse survival in our cohort of GBM patients, consistent with data from the publicly available data set Rembrandt $(10,22)$. These observations and hypotheses regarding the role of AJAP1 in GBM warrant further investigation.

Little is known about the interactions of AJAP1 except in the context of epithelial cells. In polarized epithelial cells, AJAP1 is a transmembrane protein that interacts with E-cadherin- $\beta$-catenin complexes (12), the adaptor protein complex AP-1B (20), and CD147 (16). These recent findings suggest a potential role for AJAP1 in cell-cell and cell-ECM interactions that could be involved in cell motility, migration, and invasion. As in epithelial cells and several cancers, E-cadherin and $\beta$-catenin may play a critical role in GBM cell migration as well. Modulation of the cadherin/catenin system may be facilitated by AJAP1 in GBM; however, whether and how this system interacts with AJAP1 is unknown.

Paradoxically, whereas the loss of AJAP1 expression in HeLa cells results in decreased invasiveness (16), its overexpression in glioma cells results in decreased invasiveness (13). McDonald et al also found in U251 tumor cells that AJAP1 overexpression decreased cell adhesion on ECM components and decreased migration in wound-healing assays (13), consistent with our results in stable transfections. These studies emphasize that AJAP1 may serve very different roles in different scenarios. Given these findings and our evidence of frequent loss of AJAP1 expression in GBM, we hypothesize that this phenomenon contributes to tumor cell migration in GBM as well.

During invasive migration, many cancer cells use secreted and surface-localized factors, such as matrix metalloproteinases, serine proteases, and cathepsins to proteolytically clear and remove different types of ECM substrates at their interface, including collagens, laminins, vitronectin, and fibronection. Many of these processes appear relevant to glioma cell migration as well (23). Additionally, the mechanisms required for GBM cell adhesion likely differ from those needed for active GBM migration (24). Glioma cell migration and invasion is a complex combination of multiple molecular processes, including altered tumor cell adhesion to a modified ECM, secretion of proteases, and modified actin cytoskeleton (24). Delineating the role of these processes in GBM migration and interaction with AJAP1 will require further investigation.

Other investigators have seen $A J A P 1$ deletion in brain tumors. In oligodendroglioma, McDonald et al found the specific deletion of AJAP1 in 6 of 177 (3.4\%) tumors (13), and Dong et al found its deletion in 6 of 52 (12\%) (25). The loss of loci on the distal arm of chromosome $1 \mathrm{p}$ is more frequently seen in oligodendroglioma than in GBM, where the combined loss of $1 p$ and $19 q$ is predictive of prolonged survival (26). White et al defined a single region within $1 \mathrm{p} 36.3$ that was consistently deleted in $25 \%$ of neuroblastomas, an extracranial tumor of the sympathetic nervous system commonly seen in children (27). While mapping the smallest region of a consistently deleted segment at 1p36.31 in neuroblastomas, Fujita et al found 23 genes, including AJAPl (28). Milde et al showed the loss of AJAPl in a progressively metastasizing ependymoma, another type of brain glioma (21). Our work here demonstrates that the loss of $1 \mathrm{p}$ as seen in numerous studies by us and others may be most significant because of the subsequent loss of AJAP1 on 1p. The loss of AJAP1 expression may be the critical alteration in this oncogenomic chromosomal 'hotspot' for glioblastoma and other cancers.

In this study, we extend our discovery of the frequent loss of expression of AJAP1 in GBM by demonstrating its clear role in tumor cell invasion and migration. Expression of AJAP1 in tumors results in slower tumor growth, increased survival in animal models, and correlates with survival in GBM patients. AJAP1 represents an exciting therapeutic target and prognostic factor in GBM.

Effective reduction in the extensive migration of GBM cells could greatly complement surgical resection of the primary tumor focus in this universally fatal brain tumor. Studies have already shown that glioma invasion can be the target of 
directed therapies and that these approaches may augment the efficacy of traditional therapies (29).

\section{Acknowledgements}

This material is based upon work supported by the Biomedical Laboratory Research and Development, Department of Veterans Affairs, Veterans Health Administration, Office of Research and Development. This project was supported by NINDS 5P50 NS20023, Merit Award R37 CA011898 (DDB); SPORE 5PO CA108786(DCA,DDB); NIH K12 CA100639-02, American Brain Tumor Association, Neurosurgery Research Education Foundation, National Cancer Center, and Award Number I01BX007080 from the Biomedical Laboratory Research and Development Service of the VA Office of Research and Development (DCA).

\section{References}

1. Dolecek TA, Propp JM, Stroup NE and Kruchko C: CBTRUS statistical report: Primary brain and central nervous system tumors diagnosed in the United States in 2005-2009. Neuro Oncol 14 (Suppl 5): v1-v49, 2012.

2. Kanu OO, Mehta A, Di C, Lin N, Bortoff K, Bigner DD, Yan H and Adamson DC: Glioblastoma multiforme: A review of therapeutic targets. Expert Opin Ther Targets 13: 701-718, 2009.

3. Adamson C, Kanu OO, Mehta AI, Di C, Lin N, Mattox AK and Bigner DD: Glioblastoma multiforme: A review of where we have been and where we are going. Expert Opin Investig Drugs 18: 1061-1083, 2009.

4. Stupp R, Mason WP, van den Bent MJ, Weller M, Fisher B, Taphoorn MJ, Belanger K, Brandes AA, Marosi C, Bogdahn U, et al; European Organisation for Research and Treatment of Cancer Brain Tumor and Radiotherapy Groups; National Cancer Institute of Canada Clinical Trials Group: Radiotherapy plus concomitant and adjuvant temozolomide for glioblastoma. N Engl J Med 352: 987-996, 2005.

5. Kanu OO, Hughes B, Di C, Lin N, Fu J, Bigner DD, Yan H and Adamson C: Glioblastoma multiforme oncogenomics and signaling pathways. Clin Med Oncol 3: 39-52, 2009.

6. Cancer Genome Atlas Research Network: Comprehensive genomic characterization defines human glioblastoma genes and core pathways. Nature 455: 1061-1068, 2008.

7. Matsukado Y, MacCarty CS and Kernohan JW: The growth of glioblastoma multiforme (astrocytomas, grades 3 and 4) in neurosurgical practice. J Neurosurg 18: 636-644, 1961.

8. Di C, Mattox AK, Harward S and Adamson C: Emerging therapeutic targets and agents for glioblastoma migrating cells. Anticancer Agents Med Chem 10: 543-555, 2010.

9. Mattox AK, Li J and Adamson DC: Stopping cancer in its tracks: Using small molecular inhibitors to target glioblastoma migrating cells. Curr Drug Discov Technol 9: 294-304, 2012.

10. Lin N, Di C, Bortoff K, Fu J, Truszkowski P, Killela P, Duncan C, McLendon R, Bigner D, Gregory S, et al: Deletion or epigenetic silencing of AJAP1 on 1p36 in glioblastoma. Mol Cancer Res 10: 208-217, 2012

11. Ernst A, Hofmann S, Ahmadi R, Becker N, Korshunov A, Engel F, Hartmann C, Felsberg J, Sabel M, Peterziel H, et al: Genomic and expression profiling of glioblastoma stem cell-like spheroid cultures identifies novel tumor-relevant genes associated with survival. Clin Cancer Res 15: 6541-6550, 2009.

12. Bharti S, Handrow-Metzmacher H, Zickenheiner S, Zeitvogel A, Baumann R and Starzinski-Powitz A: Novel membrane protein shrew-1 targets to cadherin-mediated junctions in polarized epithelial cells. Mol Biol Cell 15: 397-406, 2004.

13. McDonald JM, Dunlap S, Cogdell D, Dunmire V, Wei Q, Starzinski-Powitz A, Sawaya R, Bruner J, Fuller GN, Aldape K, et al: The SHREW1 gene, frequently deleted in oligodendrogliomas, functions to inhibit cell adhesion and migration. Cancer Biol Ther 5: 300-304, 2006.
14. Okawa ER, Gotoh T, Manne J, Igarashi J, Fujita T, Silverman KA, Xhao H, Mosse YP, White PS and Brodeur GM: Expression and sequence analysis of candidates for the 1p36.31 tumor suppressor gene deleted in neuroblastomas. Oncogene 27: 803-810, 2008.

15. Benjelloun A, Delavelle J, Lazeyras F and Dietrich PY: Possible efficacy of temozolomide in a patient with gliomatosis cerebri. Neurology 57: 1932-1933, 2001.

16. Schreiner A, Ruonala M, Jakob V, Suthaus J, Boles E, Wouters F and Starzinski-Powitz A: Junction protein shrew-1 influences cell invasion and interacts with invasion-promoting protein CD147. Mol Biol Cell 18: 1272-1281, 2007.

17. Gross JC, Schreiner A, Engels K and Starzinski-Powitz A: E-cadherin surface levels in epithelial growth factor-stimulated cells depend on adherens junction protein shrew-1. Mol Biol Cell 20: 3598-3607, 2009.

18. Jakob V, Schreiner A, Tikkanen R and Starzinski-Powitz A: Targeting of transmembrane protein shrew-1 to adherens junctions is controlled by cytoplasmic sorting motifs. Mol Biol Cell 17: 3397-3408, 2006.

19. Hiss JA, Resch E, Schreiner A, Meissner M, Starzinski-Powitz A and Schneider G: Domain organization of long signal peptides of single-pass integral membrane proteins reveals multiple functional capacity. PLoS One 3: e2767, 2008.

20. Resch E, Quaiser S, Quaiser T, Schneider G, Starzinski-Powitz A and Schreiner A: Synergism of shrew-1's signal peptide and transmembrane segment required for plasma membrane localization. Traffic 9: 1344-1353, 2008.

21. Milde T, Pfister S, Korshunov A, Deubzer HE, Oehme I, Ernst A, Starzinski-Powitz A, Seitz A, Lichter P, von Deimling A and Witt O: Stepwise accumulation of distinct genomic aberrations in a patient with progressively metastasizing ependymoma. Genes Chromosomes Cancer 48 (3): 229-238, 2009.

22. Cogdell D, Chung W, Liu Y, McDonald JM, Aldape K, Issa JP, Fuller GN and Zhang W: Tumor-associated methylation of the putative tumor suppressor AJAP1 gene and association between decreased AJAP1 expression and shorter survival in patients with glioma. Chin J Cancer 30: 247-253, 2011.

23. Cozad SC, Townsend P, Morantz RA, Jenny AB, Kepes JJ and Smalley SR: Gliomatosis cerebri. Results with radiation therapy. Cancer 78: 1789-1793, 1996.

24. Lefranc F, Brotchi J and Kiss R: Possible future issues in the treatment of glioblastomas: Special emphasis on cell migration and the resistance of migrating glioblastoma cells to apoptosis. J Clin Oncol 23: 2411-2422, 2005.

25. Dong Z, Pang JS, Ng MH, Poon WS, Zhou L and Ng HK: Identification of two contiguous minimally deleted regions on chromosome 1p36.31-p36.32 in oligodendroglial tumours. Br J Cancer 91: 1105-1111, 2004.

26. Brat DJ, Seiferheld WF, Perry A, Hammond EH, Murray KJ, Schulsinger AR, Mehta MP and Curran WJ; Radiation Therapy Oncology Group: Analysis of 1p, 19q, 9p, and 10q as prognostic markers for high-grade astrocytomas using fluorescence in situ hybridization on tissue microarrays from Radiation Therapy Oncology Group trials. Neuro Oncol 6: 96-103, 2004.

27. White PS, Thompson PM, Gotoh T, Okawa ER, Igarashi J, Kok M, Winter C, Gregory SG, Hogarty MD, Maris JM, et al: Definition and characterization of a region of $1 \mathrm{p} 36.3$ consistently deleted in neuroblastoma. Oncogene 24: 2684-2694, 2005.

28. Fujita T, Igarashi J, Okawa ER, Gotoh T, Manne J, Kolla V, Kim J, Zhao H, Pawel BR, London WB, et al: CHD5, a tumor suppressor gene deleted from $1 \mathrm{p} 36.31$ in neuroblastomas. J Natl Cancer Inst 100: 940-949, 2008.

29. Giese A, Bjerkvig R, Berens ME and Westphal M: Cost of migration: Invasion of malignant gliomas and implications for treatment. J Clin Oncol 21: 1624-1636, 2003.

This work is licensed under a Creative Commons Attribution-NonCommercial-NoDerivatives 4.0 International (CC BY-NC-ND 4.0) License. 